\title{
Étude de la validité interne et externe d'une échelle multidimensionnelle de Locus de contrôle spécifique aux comportements alimentaires des sportifs (LOCSCAS)
}

\section{External and internal validity of a multidimensional Locus of control scale of eating attitudes for athletes (LOCSCAS)}

\author{
Y. Paquet ${ }^{\mathrm{a}, *}$, S. Scoffier ${ }^{\mathrm{b}}$, F. d'Arripe-Longueville $^{\mathrm{b}}$ \\ a DIMPS, EA 4075, université de la Réunion, 117, rue du Général-Ailleret, 97430 Le Tampon, France \\ b Université de Nice Sophia Antipolis, LAMHES, 261, route de Grenoble, BP 3259, 06205 Nice cedex 03, France
}

\section{R É S U M É}

Les travaux en psychologie de la santé considèrent généralement le fait d'exercer du contrôle comme un facteur protecteur. Ce rôle protecteur a également été montré dans le domaine des comportements alimentaires. Cependant, les précédentes études utilisaient soit l'échelle unidimensionnelle de Rotter, soit l'échelle multidimensionnelle spécifique à la santé. Aucune échelle spécifique aux comportements alimentaires n'existe. De plus, la prise en compte des facteurs sociaux dans les précédentes échelles est limitée. En s'appuyant sur des travaux récents, l'objectif de cette étude était de proposer une échelle de locus de contrôle multidimensionnelle, prenant en compte l'environnement social du sportif et de tester sa validité interne. La validité externe de cette échelle a également été testée en relation avec les comportements alimentaires. Les résultats montrent une bonne validité interne et externe de l'échelle et confirment l'importance de la prise en compte des influences sociales. En effet, les 2 sous-dimensions "Entraîneurs, amis » et "Parents, famille " sont celles associées respectivement positivement et négativement aux troubles alimentaires.

\section{A B S T R A C T}

In the field of health psychology, the control has consistently been considered as a protective factor. This protective role has been also highlighted in eating attitudes' domain. However, current studies use the one-dimensional scale of Rotter or the multidimensional health locus of control scale, and no specific eating attitudes' scale in the sport context exists. Moreover, the social influence in previous scales is limited. According to recent works, the purpose of this study was to test the internal and external validity of a multidimensional locus of control scale of eating attitudes for athletes. One hundred and seventy-nine participants were solicited. A confirmatory factorial analysis was conducted in order to test the internal validity of the scale. The scale external validity was tested in relation to eating attitudes. The internal validity of the scale was verified as well as the external validity, which confirmed the importance of taking into consideration social influences. Indeed, the 2 subscales "Trainers, friends" and "Parents, family" are related respectively positively and negatively in eating disorders.

\footnotetext{
* Auteur correspondant.
} Adresse e-mail : yvan.paquet@univ-reunion.fr (Y. Paquet). 


\section{Introduction}

Le contrôle est un concept majeur en psychologie de la santé et a été mis en lien avec l'adoption de comportements sains et l'évitement de conduites à risque [1,2]. Le concept de contrôle est aussi relié aux désordres alimentaires [3]. Le terme de désordres alimentaires désigne l'ensemble des attitudes inadaptées liées à l'alimentation et au contrôle du poids. Ces attitudes s'étendent des restrictions sérieuses, jusqu'aux habitudes de consommation qui aident à perdre du poids ou à maintenir un corps mince [4]. Les désordres alimentaires sont une préoccupation importante pour les adolescents à laquelle les sportifs n'échappent pas [5]. Certains sports, pour lesquels la minceur est censée conférer des avantages compétitifs, seraient particulièrement "à risque » [6]. Tel est le cas des sports :

- où un faible poids est considéré comme pouvant contribuer à la vitesse et à l'efficience du mouvement (e.g., marathon, courses d'endurance);

- à catégorie de poids ;

- jugés sur des critères esthétiques qui nécessitent une connaissance de soi poussée et une morphologie spécifique.

Rascle et al. ont montré que maintenir un poids « normalement établi » nécessite des capacités personnelles à contrôler son poids [2]. L'objectif de cette étude était de valider une échelle de locus de contrôle spécifique aux comportements alimentaires chez les sportifs et d'étudier sa relation avec les attitudes alimentaires des sportifs.

\subsection{Le locus de contrôle}

Le construit de locus de contrôle (LOC) est défini à l'origine par Rotter [7] comme une expectation de contrôle traduisant le degré de représentation que possède un individu du lien entre ses comportements et/ou ses caractéristiques personnelles et les renforcements positifs ou négatifs qu'il reçoit. Dans sa conception originale [7], Rotter proposait une structure unidimensionnelle distinguant le locus de contrôle interne versus externe. Cette conception a dominé durant plusieurs années. Dans les années 1970, Levenson [8] a émis des réserves quant à la validité explicative du modèle unidimensionnel [7] et a proposé de considérer trois lieux de contrôle : interne (i.e., les renforcements obtenus étant liés à nos propres comportements), autres tout-puissants (i.e., les renforcements obtenus dépendant du comportement d'autrui significatifs) et finalement la chance (i.e., les renforcements obtenus dépendant de facteurs externes comme le hasard ou la chance). D'autres auteurs, tels Lefcourt [9] ou plus récemment, Wilkinson [10] ont confirmé que ce modèle à trois facteurs était plus explicatif et valide que le modèle unidimensionnel original de Rotter [7]. Récemment, plusieurs auteurs [11,12] ont proposé un renouvellement conceptuel suggérant la distinction de deux types d'autrui significatifs : d'une part, les "autres favorables » susceptibles d'apporter du soutien social à la situation, et d'autre part, les "autres défavorables" qui auraient davantage une influence négative par exemple sur la motivation autodéterminée [12,13]. Ces auteurs suggèrent ainsi dans différents domaines (i.e., la performance sportive et le travail) que cette distinction apporterait une meilleure prédiction de l'influence que peut exercer le locus de contrôle.

\subsection{Le locus de contrôle et comportements alimentaires}

Le concept de locus de contrôle a été mis en relation avec diverses variables telles l'anxiété $[14,15]$ ou encore la motivation $[12,13]$. Le concept de locus de contrôle est aussi un prédicteur des comportements alimentaires [1]. La majorité des travaux entre LOC et comportements alimentaires concernent la relation entre LOC et obésité. Les premières études entre LOC et obésité remontent aux années 1970, avec Gormanous et Lowe qui comparent le LOC mesuré (avec l'échelle de Rotter) de sujets obèses à celui de sujets non-obèses [16]. Ils ne trouvent à l'époque aucune différence ni entre les hommes et les femmes, ni entre les sujets obèses et non-obèses. Quelques années plus tard, Cohen et Alpert montrent une relation négative entre le score d'externalité à l'échelle de Rotter et la perte de poids [17]. L'externalité serait liée à un risque d'échec du traitement contre l'obésité. Chavez et Michaels confirment ces résultats avec une échelle spécifique à la santé [18]. En 1982, Saltzer élabore sur le modèle de Rotter une échelle spécifique pour les recherches sur l'obésité [19]. En se servant de cette échelle, Nir et Neumann [20] retrouvent les résultats antérieurs de Cohen et Alpert [17] ou Chavez et Michaels [18], indiquant que l'internalité permettrait de persévérer davantage dans un programme de régime que l'externalité. Le caractère protecteur de l'internalité est une nouvelle fois mise en avant.

Concernant le caractère prédicteur du locus de contrôle sur les comportements alimentaires, plusieurs études [21,22] se sont intéressées aux relations entre les comportements alimentaires et les comportements de contrôle du poids et le locus de contrôle relatif au comportement de santé. Les conclusions issues de ces travaux sont assez diverses car les variables utilisées, le contexte ou les sujets diffèrent. Ainsi, il est difficile de généraliser une influence particulière du locus de contrôle sur les comportements alimentaires. Par exemple, Shisslak et al. [23] montrent que les femmes boulimiques seraient davantage centrées sur un locus de contrôle externe que les femmes non boulimiques du même poids. De la même manière, Griffiths et McCabe [24] ou, plus récemment, Perry et al. [25] confirment que le locus de contrôle est un prédicteur important des désordres alimentaires.

Les différents travaux précédemment cités, bien que parfois inconsistants, révèlent une véritable relation entre le contrôle et les comportements alimentaires. Ces études utilisent en majorité soit l'échelle de Rotter (unidimensionnelle et générale), soit l'échelle multidimensionnelle spécifique à la santé (la Multidimensional Health Locus of Control Scale [26]), ou encore l'échelle de Saltzer [19], échelle spécifique à l'obésité et unidimensionnelle. Ces échelles ne sont pas adaptées aux comportements alimentaires à tendance restrictive qui caractérisent les sportifs engagés dans des disciplines "à risque » et suggèrent la nécessité de développer un outil de mesure du locus de contrôle spécifique aux comportements alimentaires chez les sportifs (LOCSCAS).

Afin de proposer un outil de mesure spécifique au contexte sportif, il paraît important de considérer l'environnement social spécifique qui le caractérise et qui est susceptible d'affecter les comportements alimentaires. L'influence de l'environnement social sur les désordres alimentaires a été mise en évidence dans la littérature en psychologie sociale [27-29]. Ces auteurs montrent une influence significative des pairs, de la famille et des médias sur les désordres alimentaires. Dans la littérature en psychologie du sport, Scoffier et al. [30] ont récemment observé une influence distincte de l'entraîneur, des amis et des parents sur les désordres alimentaires. L'entraîneur et les amis auraient une influence positive sur les désordres alimentaires, ils seraient donc vecteurs de patrons mal-adaptatifs (influence défavorable). En revanche, les parents seraient négativement reliés aux désordres alimentaires, occupant un rôle protecteur (influence favorable). Cette influence différenciée a d'ailleurs été confirmée par Scoffier, et al. en 2010 [31]. Considérant ces résultats et les travaux de Paquet et al. [12], il apparaît que l'entraîneur et les amis pourraient être assimilés aux "autres défavorables", alors que la famille et les parents pourraient être assimilés aux "autres favorables" du locus de contrôle. 
En résumé, les travaux menés en psychologie sociale montrent une relation significative entre le locus de contrôle et les désordres alimentaires [3]. Les travaux de Petrie et Greenleaf [5] suggèrent que les sportifs seraient une population particulièrement à risque concernant les désordres alimentaires. À ce jour, il n'existe pas d'outil de mesure du locus de contrôle adapté au comportement alimentaire chez les sportifs. Or, l'environnement social spécifique des sportifs et les restrictions sérieuses, ou des habitudes de contrôle du poids dans des sports où la minceur confère des avantages compétitifs, suggèrent la nécessité d'adapter l'échelle multidimensionnelle de mesure du locus de contrôle spécifique à la santé [26] au contexte du sport compétitif.

Ainsi, l'objet de cette étude était d'étudier la validité interne d'une échelle multidimensionnelle de locus de contrôle à quatre facteurs spécifiques aux comportements alimentaires dans le sport. Cette échelle permettrait de rendre compte de l'influence de deux types de "personnages tout-puissants": l'entraîneur et les amis d'un côté, la famille et les parents de l'autre. La validité externe de l'échelle a été étudiée en relation avec les désordres alimentaires, à partir des hypothèses suivantes :

- les attitudes alimentaires chez les sportifs devraient être associées négativement au locus de contrôle interne et positivement au locus de contrôle externe, comme le suggèrent les diverses études en psychologie de la santé $[1,2]$;

- les "autres favorables" (i.e., la famille et les parents) devraient être reliés négativement aux désordres alimentaires alors que les "autres défavorables" (i.e., l'entraîneur et les amis en sport) devraient l'être positivement [30].

\section{Méthode}

\subsection{Participants}

L'échantillon utilisé pour cette étude était constitué de 179 étudiants volontaires: 108 garçons et 71 filles âgés de 18 à 27 ans $\left(M_{\hat{a} g e}=21,12 ; \mathrm{ET}=2,87\right)$ pratiquant de manière régulière un sport. Les participants étaient tous français à prédominance caucasienne. Les participants ont tous répondu au questionnaire lors d'une séance de travaux dirigés. La durée de passation n'a pas excédé dix minutes. Ils ont été informés préalablement à la passation du questionnaire du fait qu'ils n'étaient pas obligés de répondre, que l'on conserverait l'anonymat et que seuls le sexe et la date de naissance étaient rapportés. Il leur a également été signalé qu'il ne s'agissait pas d'un test (i.e., il n'y avait pas de bonnes ou mauvaises réponses) et que les données obtenues ne serviraient qu'à des fins de recherches et demeureraient strictement confidentielles. Parmi ces 179 participants, 20 ont également été interrogés individuellement sur la clarté des items.

\subsection{Mesures}

Le Locus de contrôle spécifique aux comportements alimentaires chez les sportifs a été mesuré à l'aide de l'échelle multidimensionnelle de locus de contrôle specifique aux comportements alimentaires chez les sportifs (LOCSCAS) développée pour la présente étude (Tableau 1). Les différents items de l'échelle spécifique aux comportements alimentaires chez les sportifs furent créés par un comité d'experts composé de deux enseignants-chercheurs ainsi que l'auteur principal. La nouvelle échelle a été adaptée à partir de la version française de la Multidimensional Health Locus of Control Scale [26] en s'appuyant également sur le modèle à quatre facteurs de l'échelle de locus de contrôle spécifique à la performance en sport individuel [12]. La MHLCS a été choisie car elle a fait déjà l'objet de plusieurs adaptations [12,13,32]. De
Tableau 1

Items de l'échelle du Locus de contrôle spécifique aux comportements alimentaires en sports (LOCSCAS).

\begin{tabular}{|c|c|}
\hline Items & \\
\hline Interne & $\begin{array}{l}\text { 1. Concernant mon alimentation, c'est mon propre } \\
\text { comportement qui détermine si je mange de manière } \\
\text { équilibrée } \\
\text { 6. Je peux manger équilibré par mon comportement } \\
\text { 10. Si je le veux vraiment, je peux manger équilibré } \\
\text { 14. Ce qui est le plus important pour manger équilibré, } \\
\text { c'est ce que je fais moi-même } \\
\text { 20. Si j'agis de façon appropriée, je peux manger } \\
\text { équilibré }\end{array}$ \\
\hline Parents, famille & $\begin{array}{l}\text { 2. Concernant mon alimentation, avoir des contacts } \\
\text { réguliers avec mes parents, ma famille est la meilleure } \\
\text { façon pour moi de manger de manière équilibrée } \\
\text { 5. Chaque fois que je veux manger de manière } \\
\text { équilibrée, je dois attentivement écouter mes parents } \\
\text { et ma famille } \\
\text { 7. Ce qui est le plus important pour que je mange } \\
\text { équilibré, c'est ce que font mes parents et ma famille } \\
\text { 11. Les conseils que me donnent mes parents et ma } \\
\text { famille peuvent me permettre de manger équilibré } \\
\text { 16. Quand je mange équilibré, c'est en général parce } \\
\text { que mes parents et ma famille se sont occupés de moi }\end{array}$ \\
\hline Entraîneurs, amis & $\begin{array}{l}\text { 3. Si mon entraîneur et mes amis n'agissent pas de } \\
\text { façon appropriée, je ne peux pas manger de manière } \\
\text { équilibrée } \\
\text { 8. Mon entraîneur et mes amis ont beaucoup à voir } \\
\text { avec le fait que je mange équilibré ou pas } \\
\text { 12.Je peux ne pas manger équilibré de par le } \\
\text { comportement de mon entraîneur et mes amis } \\
\text { 15. Si mon entraîneur et mes amis font ce qu'il faut, je } \\
\text { peux manger équilibré } \\
\text { 18. Concernant mon alimentation, c'est le } \\
\text { comportement de mon entraîneur et de mes amis qui } \\
\text { détermine si je peux manger équilibré }\end{array}$ \\
\hline Chance & $\begin{array}{l}\text { 4. La plupart des fois où je mange de manière } \\
\text { équilibrée arrivent par chance } \\
\text { 9. La chance joue un grand rôle dans la plupart des fois } \\
\text { où je mange équilibré } \\
\text { 13. Si je ne mange pas équilibré, c'est principalement } \\
\text { dû à la malchance } \\
\text { 17. Quoi que je fasse, je ne mangerai probablement pas } \\
\text { équilibré } \\
\text { 19. Je mangerai équilibré s'il doit en être ainsi }\end{array}$ \\
\hline
\end{tabular}

manière générale, l'adaptation s'est effectuée en remplaçant les mots " maladie (ou être en bonne santé) " par les mots " manger de manière équilibrée ». Deux types d'autrui significatifs furent distingués : l'entraîneur et les amis (autres défavorables), les parents et la famille (autres favorables). L'échelle finale se compose donc de 20 items (Tableau 1), cinq pour chacune des quatre sous-échelles :

- «Interne »;

- "Entraîneurs, amis »;

- «Famille, parents »;

- "Chance».

La dimension "Interne " reflète la relation qu'établit l'individu entre ses comportements et le fait de manger ou non équilibré ; la dimension "Entraîneurs, amis » représente l'influence des comportements de ces derniers sur les comportements alimentaires des individus ; la dimension " Famille, parents » fait référence à l'influence des comportements de ces derniers sur les comportements alimentaires et finalement la dimension "Chance» illustre l'influence des facteurs externes comme la chance ou le hasard sur les comportements alimentaires. La consigne apparaissant sur le questionnaire était: " pour chacune des 20 propositions présentées ci-dessous, indiquez votre degré d'accord, en choisissant entre 4 possibilités : 
- 1 : pas du tout d'accord ;

- 2 : pas d'accord :

- 3 : d'accord ;

- 4 : tout à fait d'accord".

Attitudes alimentaires : les attitudes alimentaires ont été mesurées à l'aide de la version française de l'Eating Attitude Test (EAT) de [33], validée par Leichner et al. [34], auprès d'une population québécoise francophone. Cet instrument comporte 26 items organisés en trois sous-échelles :

- restriction alimentaire (e.g., "Je suis terrifié[e] à l'idée d'être trop gros[se]»);

- boulimie et obsession de la nourriture (e.g., "Je suis trop soucieux[se] de la nourriture »);

- contrôle de la prise alimentaire (e.g., "J'évite de manger quand j'ai faim ").

Dans la présente étude, comme c'est le cas dans d'autres travaux antérieurs [5], un indice global a été considéré, avec une consistance interne satisfaisante (rhô de Jöreskog > 75).

\subsection{Analyses}

Tout d'abord, nous avons étudié la validité de construit de l'échelle à l'aide d'une Analyse factorielle confirmatoire (AFC). Ensuite, la validité externe de l'échelle a été testée en relation avec les attitudes alimentaires. Relativement au nombre de participants et afin de maintenir un nombre de degrés de liberté acceptable, il a été décidé de réduire le nombre élevé d'indicateurs par variables manifestes (i.e., les 26 indicateurs de l'échelle EAT étaient regroupés en trois facteurs) [35]. L'AFC était basée sur 20 variables observées et quatre facteurs latents. Les analyses ont été effectuées selon la méthode de maximum de vraisemblance (maximum likelihood) à l'aide du programme LISREL $8.80^{\circ}$ [36]. Comme le recommandent plusieurs auteurs [37-39], les indices d'ajustement suivants ont été utilisés : le $\mathrm{Chi}^{2}\left(\chi^{2}\right)$, l'indice comparatif d'adéquation (Comparative Fit Index [CFI]), Non-Normed Fit Index comme indice incrémental (NNFI, [40]), les résidus standardisés (RootMean Square Error of Approximation [RMSEA]) et (RootMean Square Residual [RMR]). Les valeurs $\geq 0,90$ et $\geq 0,95$, pour le CFI et le NNFI, sont respectivement considérées comme des indices d'ajustement très satisfaisants du modèle testé [37-39]. Concernant le RMSEA et le RMR, les valeurs comprises entre 0,05 et 0,08 sont considérées comme des indices d'ajustement satisfaisants du modèle testé [37,39]. Enfin, la fiabilité a été éprouvée avec le rhô de Jöreskog [41] pour lequel des valeurs supérieures à 0,60 sont considérées comme acceptables.

\section{Résultats}

\subsection{Statistiques descriptives}

Dans un premier temps, des statistiques descriptives (i.e., moyenne, écart-type, score minimum, maximum, indices d'asymétrie et d'aplatissement) ont été réalisées pour chaque item de la LOCSCAS. Les résultats montrent que l'ensemble des scores possibles pour chaque item a été utilisé. Même si certaines moyennes semblent élevées ou faibles, elles reflètent une tendance à observer des scores élevés pour la dimension "Interne» et des scores plus faibles pour la dimension "Chance». Ainsi, on observe des moyennes aux scores de chaque item allant de 1,54 à 3,70 sur une possibilité de 4,00 et des écarts-types allant de 0,74 à 1,12. Concernant les valeurs des indices d'aplatissement et d'asymétrie (kurtosis et skewness) permettent de conclure à une distribution normale ou quasi-normale (les valeurs de l'ensemble des items sont comprises entre $-2,5$ et 2,5 ).

\subsection{Analyse factorielle confirmatoire de l'échelle locus de contrôle spécifique aux comportements alimentaires chez les sportifs}

Une AFC a été réalisée à l'aide du logiciel LISREL $8.80^{\odot}$ (Fig. 1 ) afin de tester la validité de la structure factorielle de l'échelle multidimensionnelle de locus de contrôle spécifique aux comportements alimentaires chez les sportifs (LOCSCA). Les différents indices d'ajustement $\left(\chi^{2}[179,158]=237,75, C F I=91, N N F I=90, R M R=07\right.$, RMSEA $=05$, intervalle de confiance [IC] pour le RMSEA $=040 /, 067$ ) sont conformes aux normes usuelles $[42,43]$. L'AFC permet également de montrer une relation opposant la dimension "Interne » à la dimension "Chance " $(\beta=-0,27)$ et associant les deux dimensions relatives aux autrui significatifs ("Entraîneur, amis » et "Famille, parents", $\beta=41$ ). La consistance interne de chaque facteur a été vérifiée à l'aide des Rhô de Jöreskog qui ont été calculés. Les valeurs pour l'ensemble des facteurs étaient satisfaisantes :

- 0,66 pour le locus Interne ;

- 0,75 pour la Famille et les parents ;

- 0,77 pour l'Entraîneur et amis ;

- 0,66 pour la Chance.

\subsection{Validité externe de l'échelle}

\subsubsection{Analyse de la validité de construit du modèle testé}

L'AFC met en évidence que le modèle hypothétique testé est acceptable $\left(\chi^{2}[179,214]=354,58 ; \mathrm{CFI}=87 ; \mathrm{NNFI}=0,85\right.$; $\mathrm{RMR}=0,07$; $\mathrm{RMSEA}=0,06$; intervalle de confiance [IC] pour le $\operatorname{RMSEA}=0,052 / 0,074)$. Tous les $\lambda$ s sont significatifs $(t>1,96)$.

\subsubsection{Modélisation d'équations structurales}

Afin de tester la validité externe de l'échelle, un modèle d'équations structurales a été réalisé à l'aide du logiciel LISREL $8.80^{\odot}$ permettant de tester l'influence du locus de contrôle sur les désordres alimentaires. Le modèle (Fig. 2) présente des indices d'adéquation satisfaisants $\left(\chi^{2}[179,209]=323,12, C F I=91\right.$, $\mathrm{NNFI}=90, \mathrm{RMR}=07, \mathrm{RMSEA}=06$, intervalle de confiance $[\mathrm{IC}]$ pour le RMSEA $=0,043 / 0,067$ ).

Le modèle permet de montrer, d'une part, l'influence positive qu'exercent l'entraîneur et les amis sur les attitudes alimentaires déséquilibrées, confirmant ainsi le rôle défavorable que peuvent avoir l'entraîneur ou les amis dans l'apparition de désordres alimentaires chez les sportifs. D'autre part, le rôle plutôt favorable et protecteur de la famille et des parents est également mis en avant. Ces deux résultats confirment ceux déjà trouvés par Scoffier et al. [30]. Les dimensions Interne et Chance ne sont pas reliées aux attitudes alimentaires déséquilibrées.

\subsection{Validité de construit et test-retest}

Quarante-cinq sportifs volontaires (21 garçons et 25 filles) âgés de 21 à 31 ans $\left(M_{\hat{a} g e}=26\right.$; $\left.E T=2,5\right)$ pratiquant de manière régulière, tous français à prédominance caucasienne, ont rempli dans un premier temps l'échelle de LOCSCAS. Les participants ont été recontactés 7 semaines plus tard par e-mail pour remplir à nouveau l'échelle de LOCSCAS, accompagnée de l'échelle de locus de contrôle spécifique à la santé (MHLCS [26]) et de l'échelle de locus de contrôle spécifique à la performance en sport individuel (LOCSPI [12]).

Afin de tester la validité de construit, la dimension interne et chance de l'échelle LOCSCAS ont été corrélées à celles de la MHLCS 


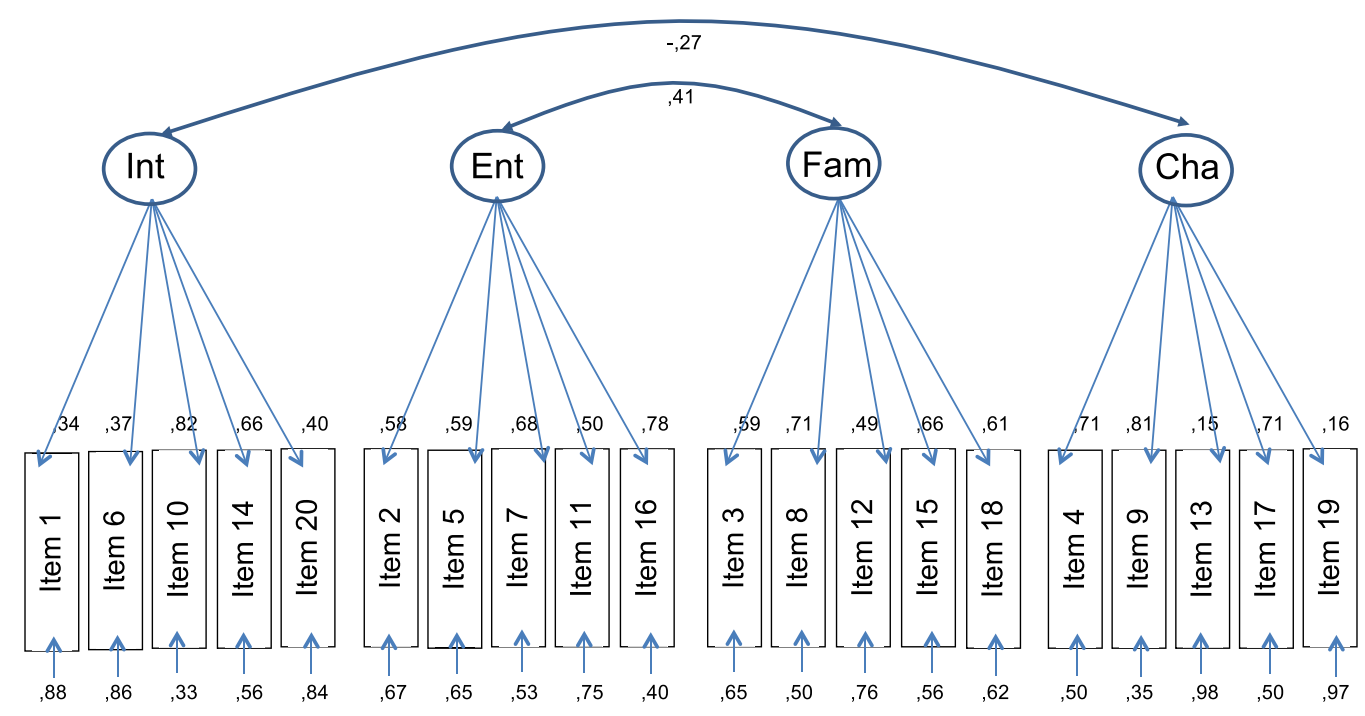

Fig. 1. Analyse factorielle confirmatoire de la LOCSCAS. Int : interne ; Ent : entraîneur ; Fam : famille ; Cha : chance.

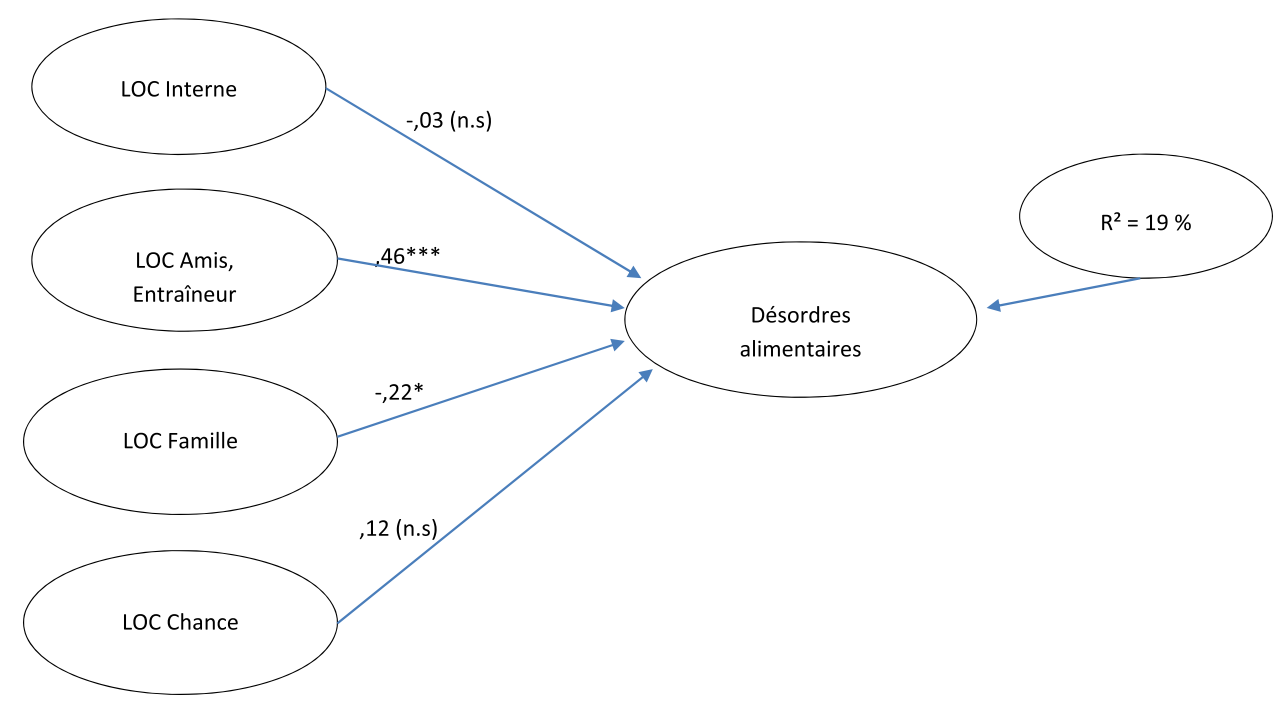

Fig. 2. Modèle de l'influence du LOC sur les désordres alimentaires. LOC: locus; $\mathrm{R}^{2}$ : pourcentage de variance expliquée : n.s. : non significatif; ${ }^{*} p<0,05 ;{ }^{* * *} p<0,001$.

et celles du LOCSPI. Les résultats montrent des relations importantes entre ces dimensions. La dimension interne du LOCSCAS étant corrélée de manière importante à celle du LOCSPI $(r=0,59$, $p<0,0001)$ et celle de la MHLCS $(r=0,73, p<0,0001)$. De même, la dimension chance du LOCSCAS est fortement corrélée à celle du LOCSPI $(r=0,77, p<0,0001)$ et celle de la MHLCS $(r=0,91$, $p<0,0001)$.

Concernant les autrui significatifs, nous nous attendions simplement à une relation entre la dimension "Entraîneur et amis " de la LOCSCAS et celle " Entraîneur » de la LOCSPI. Ce résultat est confirmé puisque les résultats montrent une relation modérée entre ces deux dimensions $(r=0,39, p<0,05)$. Les autres autrui significatifs ne sont quant à eux pas intercorrélés. Ceci est d'ailleurs logique dans la mesure où des croyances en sa famille ne sont pas en lien avec des croyances en son médecin ou encore ses adversaires.

Ces résultats montrent une bonne validité de construit à la fois divergente et convergente de l'échelle.

Concernant le test-retest, les différentes dimensions de l'échelle LOCSCAS ont été corrélées entre elles à 7 semaines d'écart. Les résultats montrent une bonne stabilité des différentes dimensions : les corrélations allant de $0,66(p<0,0001)$ pour la dimension interne à $0,87(p<0,0001)$ pour la dimension chance.

\section{Discussion}

L'objet de cette étude était d'adapter une échelle multidimensionnelle de locus de contrôle spécifique aux comportements alimentaires des sportifs et de tester sa validité interne et externe. Actuellement, les échelles les plus utilisées dans l'étude des comportements alimentaires sont celles de Rotter [7] ou encore la Multidimensional Health Locus of Control Scale (MHLCS [26]). Ces échelles ne prennent pas en compte la spécificité de l'environnement social, en particulier dans le domaine sportif. Sur la base de la nouvelle conceptualisation du locus de contrôle [12], et des travaux de Scoffier et al. [30], nous avons donc établi une échelle de LOC multidimensionnelle à quatre facteurs, dans laquelle l'entraîneur et les amis seraient considérés comme des "autres défavorables ", tandis que les parents et la famille seraient considérés comme des " autres favorables ". Ainsi, la nouvelle échelle a été adaptée à partir de la MHLCS [26] sur un modèle à quatre facteurs :

- interne ;

- autres défavorables;

- autres favorables ;

- chance. 
Une analyse factorielle confirmatoire a été réalisée afin de tester l'adéquation de nos données et du modèle théorique à quatre facteurs. Le modèle présente des indices d'adéquation corrects et conformes aux normes habituellement utilisées [42,43]. La consistance interne des différents facteurs de notre échelle a été vérifiée à l'aide des rhô de Jöreskog [41]. La structure factorielle de cette adaptation de la MHLCS est donc vérifiée.

Afin de tester la validité externe de l'échelle proposée (LOCSCAS), les relations entre les quatre facteurs de notre échelle de locus de contrôle et les attitudes alimentaires déséquilibrés ont été étudiées. Une analyse en équation structurale a été réalisée et permet de confirmer les résultats de Scoffier et al. [30]. En effet, les résultats confirment l'influence "défavorable" de l'entraîneur et des amis sur les désordres alimentaires et le rôle protecteur des parents et de la famille. Ces résultats attestent bien la validité externe de l'échelle de locus de contrôle à quatre facteurs spécifique aux comportements alimentaires des sportives proposée par dans cette étude. De plus, cela confirme l'importance de la distinction entre les " autres favorables » et les " autres défavorables » dans les études portant sur le locus de contrôle. L'absence de lien significatif entre les dimensions Interne et Chance et les comportements alimentaires soulèvent différentes questions :

- les facteurs sociaux prennent peut-être une place plus importante que les facteurs individuels?

- l'influence des dimensions Interne et Chance sur les comportements alimentaires est peut-être modérée par d'autres variables comme l'autorégulation?

En résumé, dans le domaine de la santé, comme dans celui des comportements alimentaires, les relations avec le locus de contrôle ont souvent été abordées sur le versant de l'internalité. Or l'importance de l'environnement social est loin d'être négligeable. Ainsi, comme suggéré par Levenson [8], l'ajout de la dimension d'autrui significatifs est très important. Les travaux récents [12] montrent l'importance de la distinction entre les autrui significatifs ayant une influence favorable et ceux ayant une influence plus négative. La présente étude confirme l'importance de cette distinction. De plus, cette distinction permet de montrer l'importance que peuvent jouer les parents ou la famille, probablement en termes de soutien social, face aux problèmes alimentaires rencontrés par les sportifs. Néanmoins, le rôle majeur reste celui des entraîneurs et des amis dont l'influence négative mérite encore d'être interrogée.

Plusieurs limites doivent être prises en considération en interprétant ces résultats. D'abord, les données sont des données autorapportées et ont pu avoir été influencées par la désirabilité sociale. Puis, la seconde partie de l'étude était réalisée selon une procédure transversale qui limite la stabilité dans le temps des rapports entre les variables. De plus, davantage de recherches sont nécessaires pour confirmer la validité de notre échelle sur d'autres athlètes et pour déterminer son niveau d'adaptation. D'abord, la validité du LOCSCA devrait être testée auprès d'adolescents et, au besoin, un instrument approprié selon l'âge des sujets devrait être développé. De plus, des analyses multigroupe pourraient être réalisées afin de vérifier si la structure est la même chez les filles et les garçons. Il serait également intéressant de valider cet instrument en anglais pour permettre des études transculturelles. En second lieu, la validité externe devrait être examinée en associant le locus de contrôle relatif aux comportements alimentaires des sportifs avec d'autres variables pertinentes, telles l'autorégulation des comportements alimentaires ou les styles attributionnels.

Pour conclure, l'échelle multidimensionnelle de locus de contrôle spécifique aux comportements alimentaires des sportifs paraît être un outil intéressant permettant d'appréhender les processus de contrôle des comportements alimentaires. Il s'agit ici d'une première étape de validation. Néanmoins, cette échelle spécifique possède l'avantage de tenir compte de l'environnement social du sportif (i.e., entraîneur, amis et famille) permettant une meilleure prédiction de l'influence qu'exerce le locus de contrôle sur les comportements alimentaires. Ces résultats suggèrent la mise en place de formations destinées aux entraîneurs sur les comportements à tenir face à leurs sportifs. De plus, une éducation au contrôle de leurs comportements alimentaires pourrait être développée auprès des sportifs à risque [2].

\section{Déclaration de liens d'intérêts}

Les auteurs n'ont pas précisé leurs éventuels liens d'intérêts.

\section{Références}

[1] Bruchon-Schweitzer M. Psychologie de la santé: modèles, concepts et méthodes. Paris: Dunod; 2002.

[2] Rascle N, Boujut E, Idier L. Contrôle et santé. In: Paquet Y, editor. Psychologie du contrôle : aspects théoriques et applications. Bruxelles: DeBoeck; 2009.

[3] AbuSabha R, Achterberg C. Review of self-efficacy and locus of control for nutrition and health-related behavior. J Am Dietet Assoc 1997;97:1122-32.

[4] Hobart JA, Smucker DR. The female athlete triad. Am Fam Phys 2000;61:3357-64.

[5] Petrie TA, Greenleaf CA. Eating disorders in sport: from theory to research to intervention. In: Tenenbaum G, editor. Handbook of sport psychology. 3rd ed. Hoboken, New Jersey: J. Wiley Sons, Inc; 2007. p. 352-78.

[6] Beals KA, Manore MM. Behavioral, psychological and physical characteristics of female athletes with subclinical eating disorders. Int J Sport Nutr Exerc Metabol 2000;10:128-43.

[7] Rotter JB. Generalized expectancies for internal versus external control of reinforcement. Psychol Monogr 1996;80:1-28.

[8] Levenson H. Distinctions within the concept of internal-external control: development of a new scale. Proceedings of the 80th annual convention of the. American Psychological Association; 1972. p. 261-2.

[9] Lefcourt HM. Locus of control: current trends in theory and research. $2^{\mathrm{e}} \mathrm{ed}$. Hillsdale: Lawrence Erlbaum; 1998.

[10] Wilkinson WW. The structure of the Levenson locus of control scale in young adults: comparing item and parcel indicator models. Person Individ Diff 2007;43:1416-25.

[11] Paquet Y. Les différents construits de la notion de contrôle. In: Paquet Y, editor. Psychologie du contrôle: aspects théoriques et applications. Bruxelles: DeBoeck; 2009.

[12] Paquet Y, Berjot S, Gillet N. Validation d'une échelle de locus de contrôle spécifique à la performance en sport individuel (LOCPSI). Bull Psychol 2009;62(4):351-63.

[13] Paquet Y, Lavigne GL, Vallerand RJ. Validation d'une échelle courte et multidimensionnelle de locus de contrôle spécifique au travail (MLCST). Can J Behav Sci 2014;46(1):60-5

[14] Rossier J, Rigozzi C, Berthoud S. Validation de la version française de l'échelle de contrôle de Levenson (IPC), influence de variables démographiques et de la personnalité. Ann Med Psychol 2002;160:138-48.

[15] Arnaud J, Codou O, Palazzolo J. Lien entre locus de contrôle et anxiété compétitive : étude portant sur 150 joueurs de tennis de haut niveau. Ann Med Psychol 2012;170:642-7

[16] Gormanous GK, Lowe WC. Locus of control and obesity. Psychol Rep 1975;38:30.

[17] Cohen NL, Alpert M. Locus of control as a predictor of outcome in treatment of obesity. Psychol Rep 1978;42:805-6.

[18] Chavez EL, Michaels C. Evaluation of the health locus of control for obesity treatment. Psychol Rep 1980;47:709-10.

[19] Saltzer EB. The weight locus of control (WLOC) scale: a specific measure for obesity research. J Person Assess 1982;46:620-8.

[20] Nir Z, Neumann L. Relationship among self-esteem, internal,external locus of control, and weight change after participation in a weight reduction program. J Clin Psychol 1995;51:482-90.

[21] Caggiula AW, Watson JE. Characteristics associated with compliance to cholesterol lowering eating patterns. Patient Educ Couns 1992;19:33-41.

[22] Saturnio-Springer N, Bogue N. Nutrition locus of control and dietary behavior ofpregnant women. Appl Nurs Res 1994;7:28-31.

[23] Shisslak CM, Pazda SL, Crago M. Body weight and bulimia as discriminators of psychological characteristics among anorexic, bulimic, and obese women. J Abnorm Psychol 1990;99:380-4.

[24] Griffiths JA, McCabe MP. The influence of significant others on disordered eating and body dissatisfaction among early adolescent girls. Eur Eating Disord Rev 2000;8:301-14.

[25] Perry JA, Silvera DH, Neilands TB, et al. A study of the relationship between parental bonding, self-concept and eating disturbances in Norwegian and American college populations. Eating Behav 2008;9:13-24. 
[26] Wallston KA, Wallston BS, DeVellis R. Development of the multidimensional health locus of controlscales (MHLCS). Health Educ Monogr 1978;6:160-70.

[27] Lieberman M, Gauvin L, Bukowski WM, et al. Interpersonal influence and disordered eating behaviors in adolescent girls: the role of peer modeling, social reinforcement, and body-related teasing. Eating Behav 2001;2: 215-36.

[28] Paxton SJ, Schutz HK, Wertheim EH, et al. Friendship clique and peer influences on body image concerns, dietary restraint, extreme weight-loss behaviours, and binge-eating in adolescents girls. J Abnorm Psychol 1999;108: 255-66.

[29] Shroff H, Thompson JK. The tripartite influence model of body image and eating disturbance: a replication with adolescent girls. Body Image 2006;3:17-23.

[30] Scoffier S, Maïano C, d'Arripe-Longueville F. The effects of social relationships and acceptance on disturbed eating attitudes in elite adolescent female athletes: the mediating role of physical self-perceptions. Int J Eating Disord 2010;43:65-71.

[31] Scoffier S, Paquet Y, d'Arrippe Longueville F. Effect of locus of control on disorders eating in atlhletes: the mediational role of self-regulation of eating disorders. Eating Behav 2010;11(3):164-9.

[32] Paquet Y. Validation d'une échelle de locus de contrôle spécifique à la blessure sportive. Encéphale 2008;34(2):146-52.

[33] Garner D, Olmsted M, Bohr Y, et al. The eating attitude test: psychometric features and clinical correlates. Psychol Med 1982;12:871-8.
[34] Leichner P, Steiger H, Puentes-Neuman G. Validation d'une échelle d'attitudes alimentaires auprès d'une population québécoise francophone. Rev Can Psychiatr 1994;39:49-54.

[35] Bagozzi RP, Heatherton TF. "A general approach to representing multifaceted personality constructs: application to self esteem". Struct Equat Model 1994;1:35-67.

[36] Jöreskog KG, Sörbom D. LISREL 8. 80 for Windows [Computer Software]. Lincolnwood, IL: Scientific Software International, Inc; 2006.

[37] Byrne B. Factor analytic models: viewing the structure of an assessment instrument from three perspectives. J Person Assess 2005;85:17-32.

[38] Hu L, Bentler PM. Fit indices in covariance structure modelling: sensitivity to underparametrized model misspecification. Psychol Methods 1998;3:424-53.

[39] Vandenberg RJ, Lance CE. A review and synthesis of the measurement invariance literature: suggestions, practices, and recommendations for organizational research. Organ Res Methods 2000;3:4-69.

[40] Bentler PM, Bonett DG. Significant tests and goodness of fitin the analysis of covariance structures. Psychol Bull 1980;88:588-606.

[41] Jöreskog KG. Statistical analysis of sets of congeneric tests. Psychometrika 1971;36:109-33.

[42] Kline RB. Principles and practices of structural equation modeling. New York NY: Guilford Press; 1998.

[43] Roussel P, Durrieu F, Campoy E, et al. Méthodes d'équations structurelles: recherche et applications en gestion. Paris: Economica; 2002. 